\title{
Variations in the Blood Phenytoin Levels during Long-Term Combined Treatment with S-1 and Phenytoin
}

\author{
Yutaka Negoro Takashi Higashi Hiroaki Matsuoka Kyohei Watanabe \\ Toshiaki Igarashi Yuichiro Kayano Ryoichi Yano Toshiaki Nakamura \\ Mikio Masada
}

Department of Pharmacy, University of Fukui Hospital, Fukui, Japan

\section{Key Words}

S-1 P Phenytoin · Drug interaction · Therapeutic drug monitoring

\begin{abstract}
Although combination therapy with the oral fluoropyrimidine anticancer drug S-1 and the anticonvulsant phenytoin (PHT) is known to increase blood levels of PHT and the risk of intoxication, reports on long-term monitoring of blood levels of PHT during combined S-1 and PHT treatment and a thorough understanding of their interaction are lacking. This report aims to describe interactive effects of S-1 and PHT through long-term therapeutic drug monitoring of PHT. A 72-year-old male had been prescribed oral PHT (130 mg/day) for over 20 years and started receiving S-1 therapy $(80 \mathrm{mg} /$ day for 4 weeks, followed by a 2-week rest) as postoperative adjuvant chemotherapy for gastric cancer. The blood PHT level was continuously monitored. Prior to receiving $S-1$, the patient's blood PHT concentration was 6.0 $\mu \mathrm{g} / \mathrm{ml}$, but it increased during S-1 therapy, reaching $22.9 \mu \mathrm{g} / \mathrm{ml}$ on day 84 (during a rest period of second cycle S-1 therapy). After reducing his PHT dosage to $100 \mathrm{mg} /$ day, it never reached toxic levels $(4.0-10.4 \mu \mathrm{g} / \mathrm{ml})$. It was difficult to keep blood PHT concentrations constant because of the time lag between the period of combined use of S- 1 and PHT and the timing of manifestation and disappearance of the drug interaction. The DIPS probability scale indicated a highly probable interaction between S-1 and PHT. We conclude that, when S-1 and PHT are used concurrently, occurrence and disappearance time of their interaction need to be predicted to maintain an effective and safe PHT concentration.
\end{abstract}


Negoro et al.: Variations in the Blood Phenytoin Levels during Long-Term Combined Treatment with S-1 and Phenytoin

\section{Introduction}

The antiepileptic agent phenytoin (PHT) has a narrow range of effective blood concentrations and nonlinearity pharmacokinetics. Therefore, the monitoring of its blood concentration (therapeutic drug monitoring, TDM) is essential for ensuring a proper dosage and successful treatment [1]. Because PHT is primarily metabolized by the enzymes cytochrome P450 (CYP) 2C9 and CYP2C19 [2], caution must be taken with regard to the fluctuations of the blood levels of PHT when administered in combination with other medications that induce or inhibit these enzymes. For example combination therapy with fluoropyrimidine anticancer drugs and PHT are known to increase the blood levels of PHT and the risk of intoxication [3-6].

The compound drug S-1, composed of a combination of tegafur-gimeracil-oteracil potassium, is no exception, and, in Japan, there have recently been a number of reports on drug interactions due to the combined use of S-1 and PHT [7, 8]. In these cases, patients developed PHT intoxication following more than 4 weeks of the combined treatment. Also, the S-1 treatment schedule, including periods of daily administration and interrupted by periods of rest, complicates the interpretation of its interaction with PHT. In at least 1 case, the elevated blood levels of PHT persisted even after the oral administration of S-1 had ceased [7], indicating that caution is also needed during the S-1 rest period. Unfortunately, reports on the long-term monitoring of the variations in the blood levels of PHT during the combined S-1 and PHT treatment and a thorough understanding of their interaction are lacking.

The objective of this study was to describe the interactive effects of PHT and S-1 using long-term therapeutic drug monitoring of PHT. Through regular monitoring of blood levels of PHT, we were able to conduct a pharmacokinetic analysis and describe the nature of interaction between these 2 drugs.

\section{Case Report}

The patient was a 72-year-old man with a height of $157.2 \mathrm{~cm}$, a body weight of $54.3 \mathrm{~kg}$, and a body surface area of $1.535 \mathrm{~m}^{2}$. He had been continuously treated with orally administered PHT $130 \mathrm{mg} /$ day (1.3 g/day of ALEVIATIN ${ }^{\circledR} 10 \%$ powder) for more than 20 years to prevent symptomatic epilepsy; he had never had a seizure during this time. In March 2010, he complained of upper abdominal discomfort, underwent an endoscopic examination of the upper digestive tract, and was diagnosed with gastric cancer. A pylorogastrectomy was performed at the Department of Digestive Surgery, Fukui University Hospital, and on June 9, 2010, he began outpatient postoperative chemotherapy with an oral administration of S-1 ( $80 \mathrm{mg} /$ day). One course of treatment consisted of a 28-day period of medication and a 14day off-drug period. During chemotherapy treatment, the patient continued taking other prescribed medication (pravastatin sodium, magnesium oxide, digestive enzyme complexes, itopride and sennoside).

Samples of the patient's blood were taken at every consultation and used to monitor changes in blood concentrations of PHT during the course of S-1 chemotherapy. After obtaining the patient's informed consent, PHT concentrations were measured using an automated immunoassay. The initial concentration (prior to S-1 administration of PHT) was $6.0 \mu \mathrm{g} / \mathrm{ml}$. The PHT concentration had not increased at day 14, but on day 28 , an increase was observed; by day 84 (day 14 of rest, during the second course of treatment), PHT had surpassed the toxic level ( $\geq 20 \mu \mathrm{g} / \mathrm{ml} ; 22.9 \mu \mathrm{g} / \mathrm{ml}$ ). At this time, the patient complained of 
Negoro et al.: Variations in the Blood Phenytoin Levels during Long-Term Combined Treatment with S-1 and Phenytoin

light-headedness and falls, but the third course of treatment was started without reducing the PHT dosage, and the blood concentration of PHT remained at a toxic level $(24.1 \mu \mathrm{g} / \mathrm{ml}$ at day 10 of rest during the third course of treatment, and $23.7 \mu \mathrm{g} / \mathrm{ml}$ at day 14 of rest during the third course of treatment).

The samples were also analyzed to determine blood counts and bioindicators of liver and kidney function (total bilirubin, aspartate and alanine aminotransferase, serum creatinine, and serum albumin). Initial values were as follows: total bilirubin $0.4 \mathrm{mg} / \mathrm{dl}$, aspartate aminotransferase $15 \mathrm{IU} / \mathrm{l}$, alanine aminotransferase $11 \mathrm{IU} / \mathrm{l}$, serum creatinine 0.81 $\mathrm{mg} / \mathrm{dl}$ and serum albumin $4.0 \mathrm{~g} / \mathrm{dl}$. Changes in these values were not associated with variations in the blood levels of PHT.

Regarding symptoms, falls continued, and at the beginning of the fourth course of treatment, the doses of PHT were reduced from $130 \mathrm{mg} /$ day to $100 \mathrm{mg}$ /day. As a result, at day 7 of rest during the fourth course of treatment, the blood levels of PHT decreased to 10.4 $\mu \mathrm{g} / \mathrm{ml}$, and the symptoms ceased.

While the blood concentrations of PHT continued to fluctuate after the dosage was reduced to $100 \mathrm{mg} /$ day and remained higher than those found before the initiation of S-1 therapy, they never reached toxic levels. However, the sixth course of the treatment was postponed for 2 weeks due to leukopenia. As a result, the blood concentration of PHT on the day before the initiation of the sixth course was $4.0 \mu \mathrm{g} / \mathrm{ml}$, lower than the effective blood concentration. Because the blood concentration was low, physicians increased the dosage of PHT to $130 \mathrm{mg} /$ day. The concentration of PHT at the start of the seventh course was not measured, but the symptoms of light-headedness and falls returned. Accordingly, the dosage of PHT was again reduced to $100 \mathrm{mg} /$ day. Four days later, the blood level of PHT was 18.6 $\mu \mathrm{g} / \mathrm{ml}$. Thereafter, the dosage of PHT was not modified again, but the blood concentration of PHT decreased to $10.8 \mu \mathrm{g} / \mathrm{ml}$ at day 15 of the seventh course of treatment. The variations in the blood levels of PHT are shown in fig. 1.

\section{Discussion}

While a number of reports on the drug interactions between PHT and fluoropyrimidine drugs have already been published, most of them reported having performed a TDM after the appearance of symptoms of intoxication with PHT. Also, there have been few reports on the monitoring of the blood concentrations of PHT from the time of the initiation of the combined treatment [7]. Here, we performed TDM from the initiation of S-1 treatment. Although the blood levels of PHT were found to increase after the initiation of the combined treatment, the concentrations of PHT varied in a complicated manner within the effective levels. As a result, it became difficult to determine when to reduce the dosage of PHT or to discontinue its administration, and consequently, the patient developed symptoms of intoxication. However, the results provided insights into the nature of the interaction, with regards to the timing of the increase and the decrease in the blood levels of PHT as well as the schedule of $\mathrm{S}-1$ administration.

Besides $\mathrm{S}-1$, the patient did not receive any other medication known to interact with PHT. Liver function, renal function, and serum albumin levels have been cited as factors affecting the blood levels of PHT $[9,10]$, but these do not appear to be responsible for elevated PHT levels in our patient as bioindicators (liver function - total bilirubin, aspartate and alanine aminotransferase; renal function - serum creatinine and serum albumin) of these 3 factors. Therefore, the increase in the blood levels of PHT found here was considered to be a result of interactions between S-1 and PHT. 
Negoro et al.: Variations in the Blood Phenytoin Levels during Long-Term Combined Treatment with S-1 and Phenytoin

Although the mechanism of interaction between PHT and fluoropyrimidine drugs, such as $\mathrm{S}-1$, remain unclear, in vitro studies using human liver microsomal fractions have shown that 5-FU, a metabolite of tegafur, does not directly impair the metabolism of PHT [11]. Meanwhile, in rats, the administration of 5-FU for several consecutive days has been shown to result in decreased expression and activity of CYP2C11, the equivalent of the human CYP2C9 $[12,13]$. In addition, Gunes et al. [14] reported that CYP2C9 activity decreased in patients treated with 5-FU. Based on these results, the interaction between PHT and fluoropyrimidine drugs appear to involve an indirect inhibition of PHT metabolism.

In our study, the patient's blood concentrations of PHT reached toxic levels at day 84 after the initiation of S-1 therapy. This is longer than the time required to reach similar levels in previous cases $[7,8]$. However, the increase in the blood concentrations of PHT had started at day 28. In most cases involving interactions between PHT and S-1, symptoms of PHT toxicity have occurred 4 weeks after the initiation of the combined treatment, and, even in cases where the toxic symptoms developed after a longer period of time, the increases in blood levels of PHT were first observed at 4 weeks.

The results of this study demonstrate that elevated blood levels of PHT persisted for approximately 2 weeks after the administration of S-1 ceased. At day 42 of this study (the 14th day of an S-1 rest period), the blood levels of PHT were even higher than during the previous period of S-1 administration. However, during a 28-day rest from S-1 therapy, in the fifth course of treatment, the blood levels of PHT were lower than those measured during the fifth course of S-1 administration; the low concentrations persisted even at day 10 of the sixth course of treatment. These results suggest that with prolonged rest (i.e., the 4 weeks of rest experienced here) from S-1 treatment, CYP enzymes may be restored to their original levels. These results are similar to a case of interaction between PHT and capecitabine (a fluoropyrimidine drug like S-1) [6]. In this study, the effects of capecitabine on the blood concentration of PHT persisted until day 15 after capecitabine therapy ceased, but the inhibitory effect then disappeared in a time-dependent manner. This characteristic may be common among fluoropyrimidine drugs and not limited only to $S-1$.

The above suggest that it takes more than 2 weeks until the effect of S-1 is manifested in the form of variations in the blood concentrations of PHT, and that, when S- 1 is administered for 4 weeks, the drug interaction persists for at least 2 weeks after cessation of treatment. In addition, the interaction disappears or decreases to negligible levels at 4 weeks after cessation of the oral administration of S-1.

Due to the complex interaction between PHT and S-1, determining the proper dosage of and establishing a treatment schedule for PHT is a challenge. Because PHT has a nonlinear pharmacokinetic behavior, its blood concentration can experience a rapid increase under the influence of S-1. However, if the administration of S-1 must be suspended for an extended period due to side effects such as leukopenia, the effect of S-1 on the metabolism of PHT will disappear, and the PHT dosage may become insufficient to maintain effective levels. Therefore, when S-1 and PHT are used in combination with each other, it would be preferable that PHT be replaced with another antiepileptic agent that is not a substrate of CYP2C9. In cases where S-1 and PHT must be used in combination, the possible occurrence of delayed interactions as well as the occurrence of a time lag between the period of combined administration of the drugs and the variations in the blood levels of PHT, need to be taken into consideration; TDM needs to be performed on a regular basis from the time of initiation of the combined treatment, and the doses of PHT need to be adjusted accordingly. 
Negoro et al.: Variations in the Blood Phenytoin Levels during Long-Term Combined Treatment with S-1 and Phenytoin

\section{Disclosure Statement}

There is no conflict of interest that may arise and affect the interpretation of this case report. In addition, it does not detract from the rights and benefits of patients.

\section{References}

1 Richens A: Clinical pharmacokinetics of phenytoin. Clin Pharmacokinet 1979;4:153-169.

-2 Mann MW, Pons G: Various pharmacogenetic aspects of antiepileptic drug therapy. CNS Drugs 2007;21:143164.

-3 Gilbar PJ, Brodribb TR: Phenytoin and fluorouracil interaction. Ann Pharmacother 2001;35:1367-1370.

4 Konishi H, Mosita K, Minouchi T, Nakajima M, Matsuda M, Yamaji A: Probable metabolic interaction of doxifluridine with phenytoin. Ann Pharmacother 2002;36:831-834.

$\checkmark 5$ Brickell K, Porter D, Thompson P: Phenytoin toxicity due to fluoropyrimidines (5FU/capecitabine): three case reports. Br J Cancer 2003;89:615-616.

6 Ikenishi M, Hayashiguti T, Sano M, Ueda M, Taruno Y, Ohtori T, Matsuyama K, Sasada Y, Nakatsuka E: Continually raised serum phenytoin concentration in an epileptic patient taking capecitabine undergoing cancer chemotherapy (in Japanese). Jpn J Ther Drug Monit 2011;28:109-113.

7 Tsuchishita Y, Hori S, Kameda T, Fujimura Y, Kusumoto M: Therapeutic drug monitoring of phenytoin in a patient who received tegafur: a case report (in Japanese). Jpn J Ther Drug Monit 2007;24:47-50.

-8 Tsuda A, Fujiyama J, Miki A, Hori S, Ohtani H, Sawada Y: The first case of phenytoin intoxication associated with the concomitant use of phenytoin and TS-1, a combination preparation of tegafur, gimeracil, and oteracil potassium. Cancer Chemother Pharmacol 2008;62:427-432.

-9 Itoh T, Sawada Y, Lin TH, Iga T, Hanano M: Kinetic analysis of phenytoin disposition in rats with experimental renal and hepatic diseases. J Pharmacobiodyn 1988;11:289-308.

10 Reidenberg MM, Affrime M: Influence of disease on binding of drugs to plasma proteins. Ann N Y Acad Sci 1973;226:115-126.

11 Park JY, Kim K: Inhibitory effect of 5-fluorouracil on human cytochrome P (450) isoforms in human liver microsomes. Eur J Clin Pharmacol 2003;59:407-409.

12 Stupans I, Richards DA, McClure MT: Effects of 5-fluorouracil treatment on rat liver microsomal enzymes. Xenobiotica 1995;25:1-8.

13 Afsar A, Lee C, Riddick DS: Modulation of the expression on constitutive rat hepatic cytochrome P450 isozyme by 5-fluorouracil. Can J Physiol Pharmacol 1996;74:150-156.

14 Gunes A, Coskun U, Boruban C, Gunel N, Babaoglu MO, Sencan O, Bozkurt A, Rane A, Hassan M, Zengil H, Yasar U: Inhibitory effect of 5-fluorouracil on cytochrome P450 2C9 activity in cancer patients. Basic Clin Pharmacol Toxicol 2006;98:197-200. 


\section{Case Reports in Oncology}
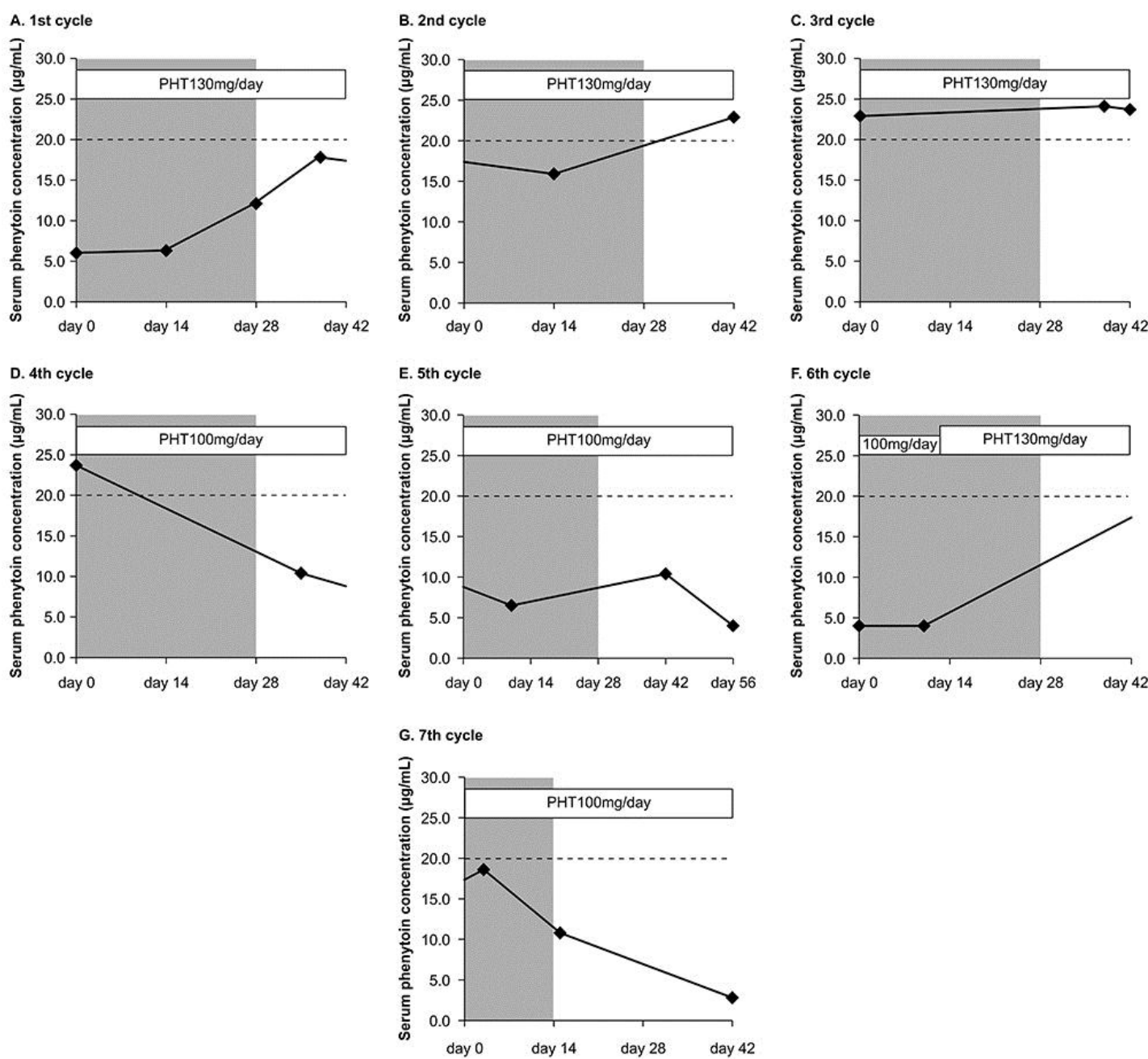

Negoro et al.: Variations in the Blood Phenytoin Levels during Long-Term Combined Treatment with S-1 and Phenytoin

(C) 2014 S. Karger AG, Basel www.karger.com/cro

Fig. 1. The time profile of serum PHT concentration and S-1 therapy. One cycle of S-1 consisted of consecutive administration for 28 days followed by 14 days of rest. The 7 th cycle lasted for 14 days. Gray column: S-1 administration). 